\title{
Del sistema integrado de gestión al sistema de gestión inteǵral: la gestión del conocimiento como estrategia en el Cuerpo Oficial de Bomberos de Bogotá"
}

\author{
From the integrated management system \\ to the comprehensive management system: knowledge \\ management as strategy in the Fire Department of Bogotá
}

Recibido: 16 de junio de 2012

Revisado: 3 de agosto de 2012

Aceptado: 21 de octubre de 2012

Olga Lucia Torres Becerra"

Martha Mercedes Suna Ladino

Convenio Universidad Santo Tomás - Icontec

\section{RESUMEN}

En Colombia, desde la promulgación de la Ley 872 de 2003, "Por la cual se crea el sistema de gestión de la calidad en la Rama Ejecutiva del Poder Público y en otras entidades prestadoras de servicios", se han implementado en las entidades del sector público sistemas de gestión, tales como el de calidad, el ambiental y el de la seguridad y salud ocupacional. Sin embargo, se evidencia un rechazo contra la implementación de estos sistemas de gestión por parte de los funcionarios, quienes obstaculizan su cabal funcionamiento afectando los procesos, las políiticas e, incluso, a las personas de las entidades.

\footnotetext{
* Artículo de investigación aplicada.

** Correspondencia: Olga Lucía Torres Becerra. Consultora en Administración Pública. Correo electrónico: olga.torresbecerra@gmail.com. Martha Mercedes Suna Ladino. Consultora en Sistemas de Gestión. Correo electrónico: martha.suna@hotmail.com.

Trabajo de grado para optar al título de Magíster en Calidad y Gestión Integral, dirigido por Ph.D. Antonio José López López.
} 
Es por ello que se asume que un sistema de gestión integral debe trascender el sistema integrado de gestión, en razón a la existencia de un eje articulador que permite la emergencia de restricciones para cada uno de sus componentes, de tal forma que todos apunten a la cohesión del sistema y, por tanto, a su organización; esta premisa se constituye en el inicio de un proceso de cambio en el caso del Cuerpo Oficial de Bomberos de Bogotá, para migrar del sistema integrado de gestión a un sistema de gestión integral, articulándolo por medio del modelo de gestión del conocimiento que se define en este trabajo.

El marco teórico se aboca fundamentalmente desde las tesis de Ikujiro Nonaka ${ }^{1}$ sobre la creación de conocimiento, de Antonio Leal Millán² sobre almacenar y recuperar el conocimiento, de Fernando Davara ${ }^{3}$ sobre transferir el conocimiento, y de Robert Grant ${ }^{4}$

1 NONAKA, Ikujiro. A dynamic theory of organizational knowledge Creation. En: Organization Science. Febrero, 1994. vol. 5, no. 1, p. 14-37. Citado por LEAL MILLÁN, Antonio. Gestión del conocimiento: fundamentos y avances en investigación [presentación, en línea], [citado en 10/06/2013]. Disponible en Internet: <http:// cmapspublic.ihmc.us/rid=1173838795854_122609230_11129/ FUNDAMENTOS.pdf>

2 LEAL MILLÁN, Antonio. Gestión del conocimiento: fundamentos y avances en investigación. [Presentación]. Disponible en Internet: <http://cmapspublic.ihmc.us/rid=1173838795854_122609230_111 29/FUNDAMENTOS.pdf>

3 DAVARA, Fernando. Conocimiento. Blog sobre innovación y tecnlogíadetendencias. TransferenciadelConocimiento[enlínea], 24 marzo de 2007. [citado en 8/06/2013]. Disponible en Internet: $<$ http://www.tendencias21.net/conocimiento/Transferencia-delConocimiento-I_a5.html>

4 GRANT, Robert M. Prospering in dynamically-competitive environments: organizational capability as knowledge integration, organization science. Julio, 1996. vol. 7, no. 4, p. 375-387. Citado por LEAL MILLÁN, Antonio. Gestión del conocimiento: fundamentos y avances en investigación [presentación, en línea], [citado en 11/06/2014]. Disponible en Internet: <en http:// cmapspublic.ihmc.us/rid=1173838795854_122609230_11129/ FUNDAMENTOS.pdf> sobre los mecanismos primarios para la aplicación del conocimiento. Igualmente, se aboca la gestión integral desde el paradigma sistémico de Edgar Morin.

Palabras clave: Gestión del conocimiento, sistemas integrados de gestión, gestión integral, gestión de la calidad, ISO 9001.

\section{ABSTRACT}

In Colombia, since the enactment of Law 872 of 2003 "By which is created the quality management system in the Executive Branch of the Public Power and other agencies that provide services", management systems have been implemented in agencies of the public sector, such as quality, environmental and security and occupational health. However, there is noticeable a rejection against the implementation of these management systems by civil servants, who hinder their proper functioning affecting the processes, the policies and even the people of the agencies.

This is why it is assumed that a comprehensive management system should transcend the integrated management system, due to the existence of a linchpin that allows the emergence of restrictions for each one of its components, so that they all point to the cohesion of the system and therefore to its organization; this premise constitutes the beginning of a process of change in the case of the Fire Department of Bogota, to migrate from the integrated management system to a comprehensive management system, articulating it through the knowledge management model defined in this work.

The theoretical framework is addressed mainly from the thesis of Ikujiro Nonaka on the creation of knowledge, of Antonio Leal Millán on storing and retrieving knowledge, of Fernando Davara on transferring knowledge, and of Robert Grant on the primary 
mechanisms for applying knowledge. Likewise, comprehensive management is addressed from the systemic paradigm of Edgar Morín.

Keywords: Knowledge management, integrated management systems, comprehensive management, quality management, ISO 9001.

\section{INTRODUCCIÓN}

Esta investigación parte de la hipótesis que afirma que un modelo de gestión del conocimiento puede asegurar la perdurabilidad de los sistemas de gestión implementados en las entidades públicas. En efecto, hay organizaciones que no solo implementan sistemas de gestión de forma independiente, sino que los integran a través de sistemas integrados de gestión (HSEQ), que unifican tanto la gestión como la administración de los subsistemas que lo componen. No obstante, las expectativas esperadas no se cumplen en muchos casos en razón a la inexistencia de un eje articulador de sus componentes y al inmediatismo por cumplir las normas que obligan a ello. Este es el caso del Cuerpo Oficial de Bomberos del Distrito Capital de Bogotá (COB), en donde la implementación del sistema integrado de gestión (HSEQ) no ha cumplido con las expectativas previstas. Lo anterior condujo a un desmejoramiento del clima organizacional en aspectos relacionados con los procesos, las personas y las políticas; así mismo, pérdida de conocimiento debido al cambio generacional y, en general, una inadecuada gestión del conocimiento.

El objetivo de la investigación es formular un modelo de gestión del conocimiento que permita articular coherentemente los sistemas de gestión de la calidad, ambiental y seguridad y salud ocupacional, y que la interacción que se genere entre ellos posibilite trascender el sistema integrado de gestión hacia un sistema de gestión integral y, por tanto, garantice su perdurabilidad.

Este estudio se enmarca en las tesis que sobre la gestión del conocimiento y, específicamente, sobre las etapas formuladas para su desarrollo - creación, almacenamiento y recuperación, transferencia y aplicación- formulan, entre otros, Ikujiro Nonaka ${ }^{5}$ sobre la creación de conocimiento, Antonio Leal Millán sobre almacenar y recuperar conocimiento ${ }^{6}$, Fernando Davara ${ }^{7}$ sobre transferir conocimiento, y Robert $\mathrm{Grant}^{8}$, quien propone mecanismos primarios para la aplicación del conocimiento. Por último, se aboca la construcción del modelo de gestión integral desde el paradigma sistémico de Edgar Morín ${ }^{9}$, el cual facilita, a través de las etapas de la gestión del conocimiento, establecer una adecuada articulación —o ciudadanía sistémica - entre los subsistemas que lo conforman.

Hablar de un sistema de gestión integral implica fundamentarse en el paradigma sistémico propuesto por Morín, el cual trasciende la teoría general de sistemas en la que se apoyan los sistemas integrados de gestión. En el paradigma sistémico, el todo es una macrounidad en la que las partes no se fundan con él, como en el tronco del árbol de tres ramas con el que se identifican los sistemas integrados, sino que las partes o subsistemas tienen una doble identidad: una propia que permanece en ellas y que, por tanto, no se reduce al todo, y una identidad común, la de su ciudadanía sistémica ${ }^{10}$; esto constituye el principio de la emergencia.

5 NONAKA. Op. cit.

6 LEAL. Op. cit.

7 DAVARA. Op. cit.

8 GRANT. Op. cit.

9 MORIN, Edgar. Ciencia con conciencia: por un paradigma de complejidad. Barcelona: Antrophos, 1994. p. 341-369.

10 MORÍN, Op. cit., p. 341-369. 
Las características principales de la propuesta de Morín son: a) la mayor parte de los sistemas no están conformados por partes, elementos o constituyentes, sino por acciones entre unidades complejas cuyo conjunto constituye la organización del sistema; b) la problemática del sistema no se resuelve con la relación todo/partes (TGS) ni con la reducción de las ideas sistémicas al todo (holismo), sino que estas relaciones deben estar mediatizadas por el término interacciones y por el principio de emergencia ya descrito. El principio de las restricciones establece que toda relación sistémica ejerce restricciones sobre los elementos que la integran, mediante reglas, jerarquías, subordinación de los componentes al todo, especializaciones, estabilidad y dispositivos de regulación y control (Cunha, 1989); es aquí donde, respecto de este estudio, las etapas de la gestión del conocimiento ejercen restricciones y articulan los elementos del HSEQ.

Desde esta perspectiva teórica es que se aboca la construcción de un modelo de gestión de conocimiento que permita articular coherentemente cada uno de los subsistemas implementados en el COB.

\section{METODOLOGÍA}

La investigación se desarrolla desde el paradigma epistemológico positivista ${ }^{11}$, que busca, a partir de la observación y la aplicación de instrumentos cuantitativos y cualitativos, establecer relaciones para identificar el papel de un modelo de gestión del conocimiento para la articulación de los subsistemas de un sistema integral de gestión en el Cuerpo Oficial de Bomberos de Bogotá (COB), que garantice la perdurabilidad de

11 LÓPEZ LÓPEZ, Antonio José. Taller de tutoría de proyectos. Fortalecimiento de competencias investigativas. Cohorte 12. Maestría Calidad y Gestión Integral [presentación]. Bogotá, Colombia: Convenio USTA-ICONTEC, 2011. Diapositivas 7-19. los sistemas que conforman el sistema. En este orden de ideas, el método por aplicar es inductivo-deductivo; esto significa conocer la realidad desde lo particular hacia lo general. Desde este enfoque, la investigación tiene sucesivamente los siguientes momentos: planificación, ejecución, evaluación y comunicación de los resultados.

Sin embargo, apoyada en el método positivista y, más exactamente, neopositivista ${ }^{12}$, esta investigación no pretende el descubrimiento de leyes generales ${ }^{13}$ o universales, sino la construcción de conocimiento a partir de la racionalidad y la lógica, sobre el aporte que un modelo de gestión del conocimiento pueda ejercer a la perdurabilidad del sistema de gestión integral, y que trascienda el sistema integrado de gestión vigente.

La investigación se apoya en información primaria y secundaria, tales como investigaciones ya elaboradas y el estudio de clima organizacional realizado en el COB; asimismo, en una encuesta a través de la cual se identifica información de los aspectos críticos en cada una de las etapas de la gestión del conocimiento y la obtención de resultados de los indicadores establecidos.

El diseño metodológico incluye un estudio piloto para verificar las bondades de los instrumentos por aplicar, el cual se realiza en forma previa al trabajo de campo. La investigación tiene las características de un estudio de caso, porque se asume como una "estrategia metodológica de investigación científica, útil en la generación de resultados que posibilitan el fortalecimiento, crecimiento y desarrollo de las teorías

12 ATENCIA, José María; GAVILÁN, Juan y RODRÍGUEZ, Agustín. Iniciación a la historia de la filosofía. 2 ed. Málaga, España: Editorial Librería Ágora, 1993. 409 p.

13 Ibid., p. 268. 
existentes" 14 , lo cual es perfectamente congruente con el paradigma positivista desde el cual se asume. En este sentido, se apoya en las teorías sobre gestión del conocimiento y, por lo tanto, contribuye al desarrollo de un campo científico determinado.

El método utilizado en la investigación es inductivo, entendido como la observación de fenómenos particulares para identificar casos generales ${ }^{15}$, de acuerdo con las siguientes etapas: a) una de observación, descripción y reflexión del fenómeno para la generación de las hipótesis, utilizando enfoques cualitativos y cuantitativos a partir de información primaria, con el fin de explicar el comportamiento, las causas y los efectos del fenómeno; b) una segunda consistente en la comprobación del fundamento de la hipótesis a través de enfoques cuantitativos; c) una tercera que implica la construcción de una propuesta de modelo por seguir en organizaciones similares, elaborada a partir del análisis lógico racional de la información obtenida.

De acuerdo con los interrogantes establecidos en la definición del problema y con los objetivos planteados, se establece la siguiente hipótesis para el proyecto de investigación:

El diseño de un modelo de gestión del conocimiento, de forma tal que permita la interacción y articulación de los sistemas implementados en el sistema integrado de gestión del $\mathrm{COB}$ de Bogotá, garantiza la iniciación de un proceso que permite trascender del sistema integrado de gestión al sistema de gestión integral en esa organización.

14 MARTÍNEZ CARAZO, Piedad Cristina. El método de estudio de caso. Estrategia metodológica de la investigación científica. En: Pensamiento y Gestión. 2006. no. 20, p. 189.

15 LÓPEZ LÓPEZ, Op. cit.
El universo lo constituyen todos los miembros del $\mathrm{COB}$, quienes suman un total de 763 funcionarios. Pese a que la unidad de análisis es simple, pues se analiza toda la entidad, se aplican instrumentos metodológicos a subunidades de análisis definidas ${ }^{16}$, como las 17 estaciones de bomberos y la sede administrativa.

Para determinar la muestra se utiliza un muestreo aleatorio estratificado con afijación proporcional. Este tipo de muestreo se utiliza cuando se dispone de información sobre las características de la población por encuestar y evita que alguno de los grupos quede menos representado.

El análisis de las encuestas se hace por medio del software Statistical Package for the Social Sciences (SPSS), versión 17.0, el cual permite la obtención de estadísticas descriptivas y tablas de contingencias.

El instrumento utilizado en el desarrollo de la investigación es la encuesta ${ }^{17}$, que se elabora teniendo en cuenta la forma cómo se presenta cada una de las etapas de la gestión del conocimiento en la actualidad y cómo deberían ser, basándose en ejemplos de la realidad del COB. Este instrumento se aplica en el desarrollo del trabajo de campo a través de preguntas estructuradas, realizadas de manera personal de acuerdo con la muestra y la unidad de análisis determinada.

16 Ibid., pp. 183-186.

17 UCH-RRHH el portal de estudiantes de RRHH. Concepto de encuesta [en línea], 2006 [citado en 2006-06-26]. Disponible en Internet: <http:// www.gestiopolis.com/recursos/documentos/ fulldocs/mar/tipencuch. htm > 


\section{RESULTADOS Y DISCUSIÓN}

Dado que la investigación pretende diseñar un modelo de gestión del conocimiento para el COB de Bogotá, que permita la cohesión de los distintos subsistemas que conforman el vigente sistema integrado de gestión, se hace necesario estructurar una herramienta que contribuya a una adecuada recolección de la información. Para ello se elabora una matriz categorial que resulta de la interrelación e integración de los conceptos y teorías seleccionadas respecto de las etapas de la gestión del conocimiento y su adecuada o inadecuada presencia en el interior de las normas de los subsistemas de gestión de la calidad, gestión ambiental y gestión de seguridad y salud ocupacional.

Esta matriz categorial se desarrolla en diferentes fases: primero, como fruto del análisis del marco teórico, se decide tomar cuatro de las teorías analizadas como eje de construcción; segundo, a partir de la identificación de las etapas de la gestión del conocimiento como categorías relevantes, se procede a establecer su interrelación con los conceptos derivados de esas teorías; la relevancia se encuentra directamente relacionada con el peso específico de cada categoría respecto de su impacto sobre la gestión integral y la adecuación de dichos conceptos al contexto normativo de los subsistemas que lo conforman. Las cuatro categorías establecidas corresponden a la concepción del grupo de trabajo, aporte de la teoría, etapas de la gestión del conocimiento y requisitos de las normas correspondientes a cada uno de los subsistemas; finalmente, se establecen los indicadores y se definen los instrumentos que permiten medir las diferentes etapas de la gestión del conocimiento.

En la matriz categorial, la fila denominada concepto integrador surge como resultado del análisis e interpretación de las categorías indicadas en la matriz, sobre la base del conocimiento y experiencia de las investigadoras, constituyendo así un aporte y perspectiva novedosos sobre los que se basa la construcción de indicadores que facilitan el diseño del modelo de gestión integral emergente.

Se presentan a continuación los resultados de la construcción de indicadores para medir la gestión del conocimiento en sus diferentes etapas; ellos son:

- Indicadores de la creación del conocimiento. Para esta primera etapa, en sus cuatro formas, socialización, combinación, internalización y externalización, se establecen los indicadores que permitan medir cómo perciben los bomberos la creación del conocimiento en la entidad y cómo creen que se debe crear ese conocimiento:

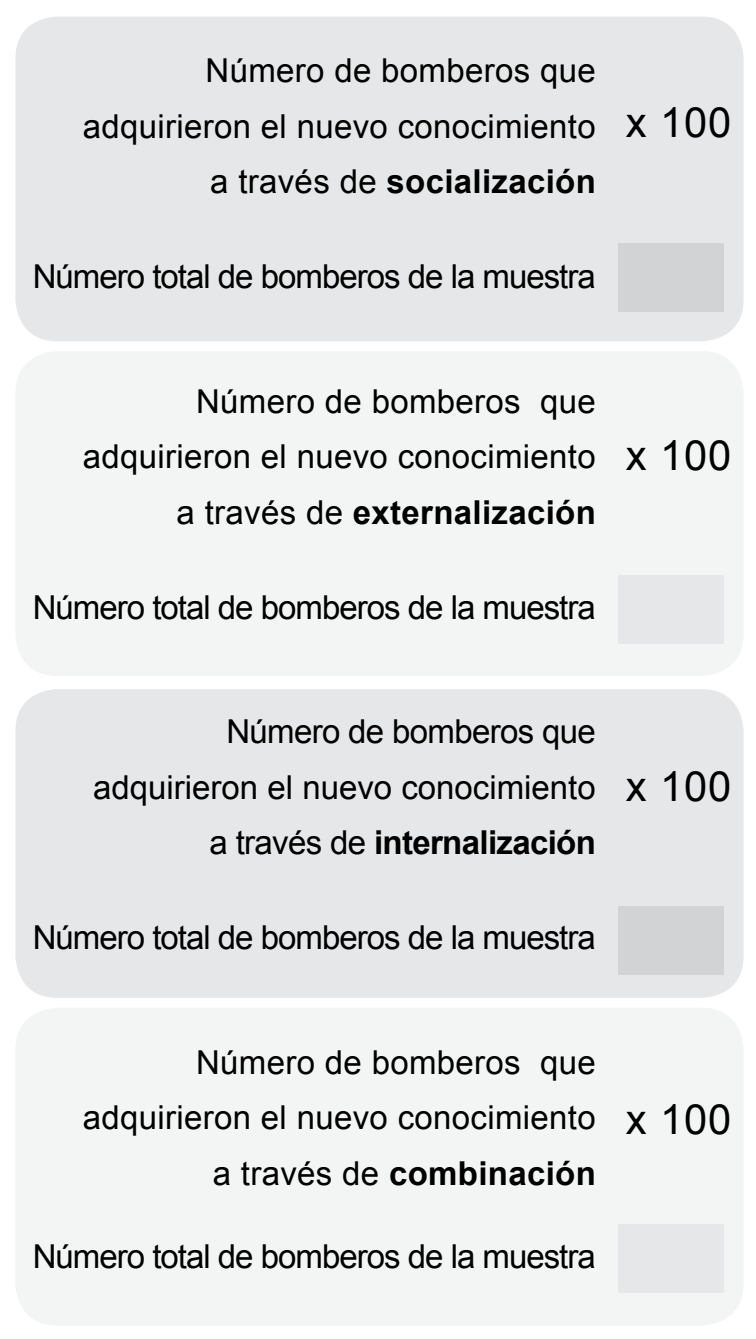


Número de bomberos que creen que la forma más eficaz para

la adquisición del conocimiento $x 100$ sería por socialización

Número total de bomberos de la muestra

Número de bomberos que creen que la forma más eficaz para

la adquisición del conocimiento $x 100$ sería por externalización

Número total de bomberos de la muestra

Número de bomberos que creen que la forma más eficaz para la adquisición del conocimiento $x 100$ sería por internalización

Número total de bomberos de la muestra

Número de bomberos que creen que la forma más eficaz de adquirir x 100 el conocimiento sería por combinación

Número total de bomberos de la muestra)

- Indicadores de almacenamiento y recuperación del conocimiento. Para la segunda etapa, en las cuatro formas establecidas, hardware, software, trabajo en colaboración y herramientas inteligentes, se establecen los indicadores que miden cómo perciben los bomberos en la actualidad la forma cómo se almacena y recupera el conocimiento en la entidad y cómo creen que se debe recuperar y almacenar el conocimiento generado en la entidad:
Número de bomberos que consideran que el hardware es el medio más importante para almacenar $x 100$ y recuperar el conocimiento

Número total de bomberos de la muestra

Número de bomberos que consideran que el software es el medio más importante para almacenar $\times 100$ y recuperar el conocimiento Número total de bomberos de la muestra

Número de los que creen que el trabajo en colaboración es el mejor medio para almacenar y recuperar

el conocimiento

Número total de bomberos de la muestra

Número de los que creen que las herramientas inteligentes son el mejor medio para almacenar

y recuperar el conocimiento

Número total de bomberos de la muestra

Número de los que creen que el hardware sería el medio más importante para almacenar x 100 y recuperar el conocimiento

Número total de bomberos de la muestra 
Número de bomberos que creen

que el software sería el medio más importante para almacenar

y recuperar el conocimiento

Número total de bomberos de la muestra

Número de los que creen que el trabajo en colaboración sería el mejor medio x 100 para almacenar y recuperar el conocimiento

Número total de bomberos de la muestra

Número de los que creen que las herramientas inteligentes serían el mejor medio para almacenar $x 100$ y recuperar el conocimiento

Número total de bomberos de la muestra

- Indicadores de transferencia del conocimiento. Para la tercera etapa, en las tres formas establecidas, endógeno, exógeno y combinado, se establecen los siguientes indicadores, los cuales miden cómo perciben los bomberos en la actualidad la forma cómo se trasfiere el conocimiento en la entidad y cómo creen que se debe transferir el conocimiento que se genera en la entidad:

Número de bomberos que consideran el modelo endógeno como el que $\times 100$ más ha contribuido al aprendizaje

Número total de bomberos de la muestra
Número de bomberos que consideran el modelo exógeno como el que $\times 100$ más ha contribuido al aprendizaje

Número total de bomberos de la muestra

Número de bomberos que consideran el modelo combinado como el que $\times 100$ más ha contribuido al aprendizaje

Número total de bomberos de la muestra

Número de bomberos que consideran el modelo endógeno como el que $\times 100$ más contribuiría al aprendizaje

Número total de bomberos de la muestra

Número de bomberos que consideran el modelo exógeno como el que $\times 100$ más contribuiría al aprendizaje

Número total de bomberos de la muestra

Número de bomberos que consideran

el modelo combinado como el que $\times 100$ más contribuiría al aprendizaje

Número total de bomberos de la muestra 
- Indicadores de la aplicación del conocimiento. Para la cuarta etapa de la gestión del conocimiento, en las tres formas establecidas, directrices, rutinas organizativas y equipos autónomos de tarea, se establecen los siguientes indicadores, los cuales miden la forma en que perciben los bomberos en la actualidad cómo se aplica el conocimiento generado en la entidad:

Número de bomberos que aplican las directrices en el desarrollo $\times 100$ de las actividades

\section{Número total de bomberos de la muestra}

Número de bomberos que aplican las rutinas organizativas en $\times 100$ el desarrollo de las actividades

Número total de bomberos de la muestra

\section{Número de bomberos que aplican \\ los equipos autónomos de tarea $\times 100$ en el desarrollo de las actividades}

\section{Número total de bomberos de la muestra}

Una vez aplicada la encuesta, se identifica el desarrollo de las etapas del modelo de gestión del conocimiento en el Cuerpo Oficial de Bomberos de Bogotá, detectando las falencias de cada etapa para considerarlas en el diseño del sistema de gestión de conocimiento, aspecto fundamental de la presente investigación.

Para obtener resultados acordes a cada una de las etapas de la gestión del conocimiento, en las preguntas planteadas en la encuesta, se dieron opciones de respuesta de acuerdo con los instrumentos que se manejan en la entidad. Por tanto, la gestión del conocimiento, dentro de sus cuatro etapas, se desarrolla en el COB en la actualidad como se muestra en la figura 1.

Figura 1. Diagnóstico de las cuatro etapas de la gestión del conocimiento COB

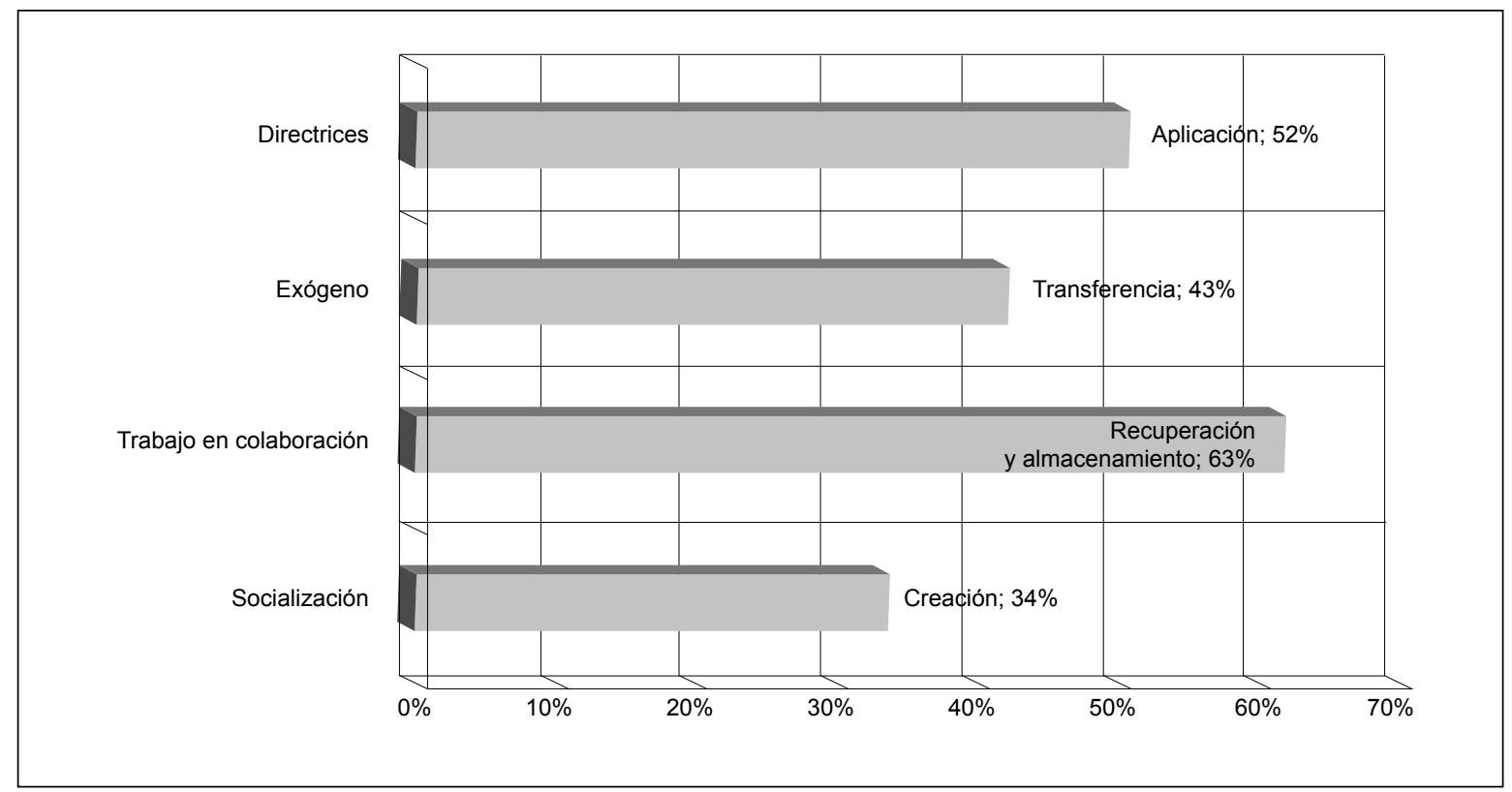

Fuente: Elaboración de las autoras con base en los resultados de las encuestas. 
De acuerdo con estos resultados, cada una de las cuatro formas ha contribuido para la implementación del SIG del Cuerpo Oficial de Bomberos de la siguiente manera:

En la etapa de creación de conocimiento: socialización, con la enseñanza rutinaria de sus compañeros, ha consolidado la información para el levantamiento de toda la documentación: manuales, procesos, procedimientos, etc., apoyada con la internalización que se encuentra en segundo lugar, mediante los simulacros, movilizaciones, capacitaciones internas, que permiten el mejoramiento de la documentación existente, para la generación de todo el conocimiento que en la actualidad sustenta el sistema integrado de gestión que se tiene implementado en el COB. Esto corrobora uno de los principales obstáculos en la construcción de sistemas de gestión perdurables en la cultura organizacional, y es la fuerte influencia de los funcionarios más antiguos, quienes presentan gran resistencia al cambio, especialmente frente a la documentación y evaluación de los sistemas de gestión.

En la etapa de recuperación y almacenamiento del conocimiento: el trabajo en colaboración, con la minuta de guardia, los archivos físicos y las actas de reunión, mantiene el conocimiento generado en la implementación del SIG para su consulta y aplicabilidad posterior. Sin embargo, este resultado muestra la fuerte resistencia a la implementación de nuevas tecnologías de la información y las comunicaciones y el gran esfuerzo que el cambio conlleva.

En la etapa de transferencia del conocimiento: el modelo exógeno es el más aplicable mediante las capacitaciones teórico-prácticas en la academia, la capacitación internacional, la formación de brigadas y en la atención de los servicios, en donde los de menor experiencia se apoyan en el personal más experimentado para adquirir los conocimientos necesarios para el desarrollo de las actividades de atención de incidentes; todo esto apoyado con el modelo endógeno y las capacitaciones internas sobre los procedimientos. Este resultado es la manifestación de una tendencia en el interior del COB a considerar el conocimiento como algo acabado y, por otra parte, estar dispuestos siempre a impartir instrucción y entrenamiento.

Finalmente, en la etapa de aplicación del conocimiento: las directrices son el mecanismo al cual acude el personal operativo del COB para realizar sus actividades en la atención de los incidentes, tales como los procedimientos, las políticas y las instrucciones. Así mismo, se apoyan mucho en la aplicación de los protocolos y en las rutinas establecidas.

Se puede concluir que en el Cuerpo Oficial de Bomberos existen mecanismos que dan cuenta de cada una de las etapas de la gestión del conocimiento, los cuales evidencian fuertes inercias culturales que se constituyen en barreras para la perdurabilidad de los sistemas de gestión implementados, denotando la ausencia de elementos de cohesión que permitan pasar de un sistema de gestión integrado a un sistema de gestión integral.

Por esto es importante conocer cómo, según la creencia del personal de la entidad, debería ser cada etapa y así establecer un modelo de gestión del conocimiento con aspectos que permitan la cohesión y la perdurabilidad necesaria, para que la implementación de estos sistemas no afecte a la entidad en ningún sentido y no se convierta en un obstáculo, sino que, por el contrario, contribuya a la construcción de un sistema de gestión integral.

A partir de la información de la encuesta anterior, en la cual también se plantean preguntas para conocer cómo debería ser cada etapa de la gestión del conocimiento, se formula una propuesta de modelo de gestión de conocimiento que sirva de eje articulador de los sistemas que conforman el actual sistema de gestión del COB de Bogotá, aplicado a sus procesos, las políticas y las personas, y las estructuras básicas del sistema actual.

En la figura 2 se presenta el resultado para el planteamiento del modelo. 
Figura 2. Modelo de las cuatro etapas de la gestión del conocimiento, COB

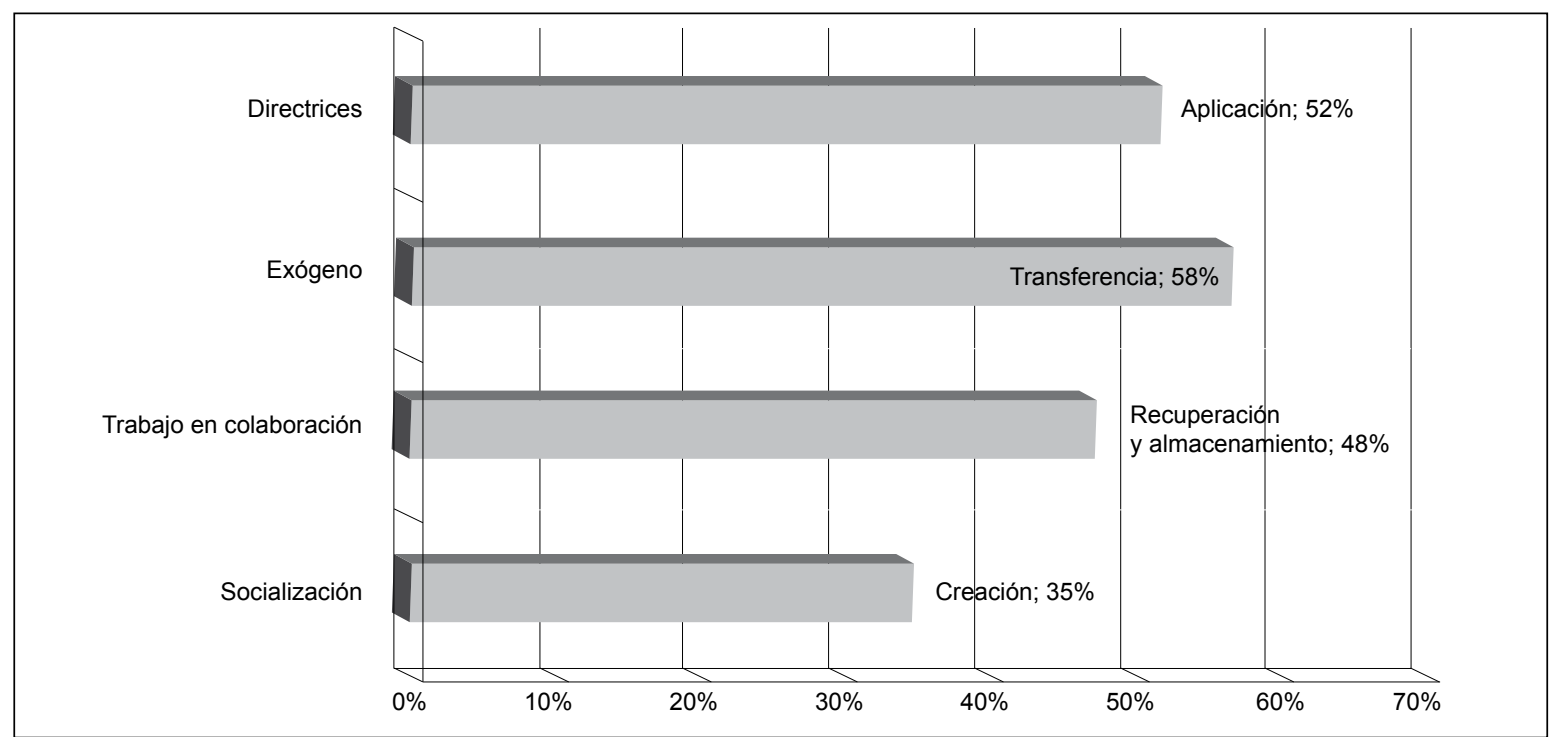

Fuente: Elaboración de las autoras con base en los resultados de las encuestas aplicadas.

De acuerdo con lo anterior, se deduce que el personal prefiere, en la etapa de creación del conocimiento, pasar de la socialización (de la experiencia y enseñanza rutinaria de sus compañeros) a una creación más especializada a través de ejercicios y de procedimientos documentados, lo cual es destacable. Las demás etapas continúan de la misma manera que en el diagnóstico, lo cual supone al menos tres frentes de trabajo relevantes, a saber: gestión del cambio, mejoramiento de las competencias cognitivas, énfasis en las tecnologías de la información y las comunicaciones.

En atención a estos resultados, se puede deducir que cada una de las cuatro formas debería contribuir para la perdurabilidad del SIG del Cuerpo Oficial de Bomberos de la siguiente manera:

En la etapa de creación de conocimiento: internalización y combinación, con los simulacros, movilizaciones, capacitaciones internas, así como las simulaciones, los planes específicos de respuesta (PER) y la formación externa, para la generación del conocimiento que debería soportar el sistema de gestión integral del COB.
En la etapa de recuperación y almacenamiento del conocimiento: el trabajo en colaboración, con la minuta de guardia, los archivos físicos y las actas de reunión, tiene una importancia sobresaliente frente a las demás herramientas. Por ello se requiere un esfuerzo importante en tecnologías de la información y de las comunicaciones.

En la etapa de transferencia del conocimiento: el modelo exógeno es el que debería aplicarse mediante las capacitaciones teórico-prácticas en la academia, la capacitación internacional, la formación de brigadas y en la atención de los servicios. Este modelo es ideal, siempre que el modelo endógeno esté consolidado, es decir, que la formación integral del bombero esté consolidada.

Finalmente, en la etapa de aplicación del conocimiento: las directrices son el mecanismo al cual debe acudir el personal operativo del COB para realizar sus actividades y en la atención de los incidentes, tales como los procedimientos, las políticas y las instrucciones. Sin embargo, no desconoce la importancia de la aplicación de los protocolos y las rutinas establecidas. Al respecto, debe fortalecerse el conocimiento experto. 
En este sentido, el modelo de gestión del conocimiento que permita la perdurabilidad del sistema de gestión integral en el Cuerpo Oficial de Bomberos de Bogotá tiene las características que a continuación se relacionan, y que permiten trascender de un sistema integrado de gestión a un sistema de gestión integral:

\section{A. Implementación de un SGC por etapas simultáneas}

Se deben implementar simultáneamente las etapas de la gestión del conocimiento, a saber:

- Creación de conocimiento. Con un énfasis marcado en la internalización y combinación, dando una significativa menor importancia a los procesos de socialización. Es decir que se valora significativamente el paso de un conocimiento tácito a uno explícito y documentado. Este tal vez sea uno de los cambios más importantes en todas las etapas de la gestión del conocimiento en el $\mathrm{COB}$, dado el grado de madurez de los sistemas implementados y la cultura organizacional y que es fundamental para la transformación de sistemas integrados a sistemas integrales.

- Almacenamiento y recuperación. Se mantiene el énfasis en el trabajo en colaboración, sin embargo debe destacarse que, dada la cultura organizacional, hay una inercia que se constituye en una barrera para el uso intensivo de las tecnologías de la información y de las comunicaciones, por lo que esta etapa debe hacer un intensivo entrenamiento y aplicación de tales tecnologías, resaltando que esta puede ser la principal barrera para la perdurabilidad de los sistemas de gestión, así como para la trasformación en sistemas de gestión integrales.

- Transferencia. Esta etapa debe intensificar la transferencia exógena, considerada un gran avance que permitirá mayor apertura a nuevos conocimientos e intercambio de experiencias, y afianzarála mejora continua de los sistemas de gestión implementados y, por consiguiente, su mayor perdurabilidad. Sin embargo, no se debe descuidar la transferencia endógena como proceso de formación continuo en el interior del COB.

- Aplicación. Por su parte, las directrices de expertos, las rutinas organizativas y los equipos autónomos de tareas mantienen su relevancia y el papel preponderante en la organización y en la respuesta a incidentes. Es recomendable, para una labor técnica y especializada y de alto riesgo, como el servicio bomberil, fortalecer los equipos autónomos de tarea privilegiándolos sobre las directrices, ya que estos fundamentan la autonomía en la experticia.

\section{B. Coherencia y alineación con los sistemas integrados}

Para garantizar el papel articulador del modelo de gestión del conocimiento para los demás sistemas de gestión, es necesario asegurarse de la alineación de los tres sistemas implementados — calidad, seguridad y salud ocupacional, ambiental—en, por lo menos, los siguientes aspectos:

- Planificación.

- Identificación de requisitos y características.

- Alcance.

- Política y objetivos.

- Competencia del personal.

- Cultura y toma de conciencia.

- Comunicación interna.

- Documentación y registros.

- Mejora continua: acciones correctivas, preventivas, auditorías, revisión por la dirección.

- Plan de respuesta a emergencias: producto no conforme.

\section{Equilibrio entre las políticas, los procesos y las personas}

Para la perdurabilidad de los sistemas de gestión, es fundamental que las políticas y los procesos sean el 
resultado de un modelo de gestión del conocimiento soportado en las competencias de las personas, las cuales deben ser continuamente desarrolladas. Este último aspecto es importante para que la participación de expertos no se asuma desde la imposición sino desde la comprensión, desarrollando una adecuada cultura de gestión del cambio desde el fortalecimiento de estas competencias.

\section{Implementación orientada al aprendizaje organizacional}

Del mismo modo que un sistema de gestión de calidad está orientado a la satisfacción del cliente, uno de seguridad y salud ocupacional se orienta a la reducción de riesgos, y un sistema de gestión ambiental se dirige a la reducción de impactos ambientales, el sistema de gestión del conocimiento debe estar orientado al aprendizaje organizacional para procurar la satisfacción del cliente, la reducción de los riesgos y de los impactos ambientales en su conjunto, a partir del mejoramiento de las competencias de los funcionarios.

\section{E. Articulación con los procesos de inducción y capacitación}

La gestión del conocimiento y su relación con los sistemas de gestión debe ser aspecto fundamental para elaborar los procesos de inducción y los planes institucionales de capacitación anuales, a fin de lograr coherencia y un óptimo afianzamiento en la cultura organizacional.

En la figura 3 se presenta el esquema del modelo de gestión del conocimiento y su alineación con los requisitos de los subsistemas del sistema de gestión integral: calidad, ambiental y de seguridad y salud ocupacional, para su respectiva aplicación o implementación.

Figura 3. Modelo de gestión del conocimiento para $\mathrm{COB}$

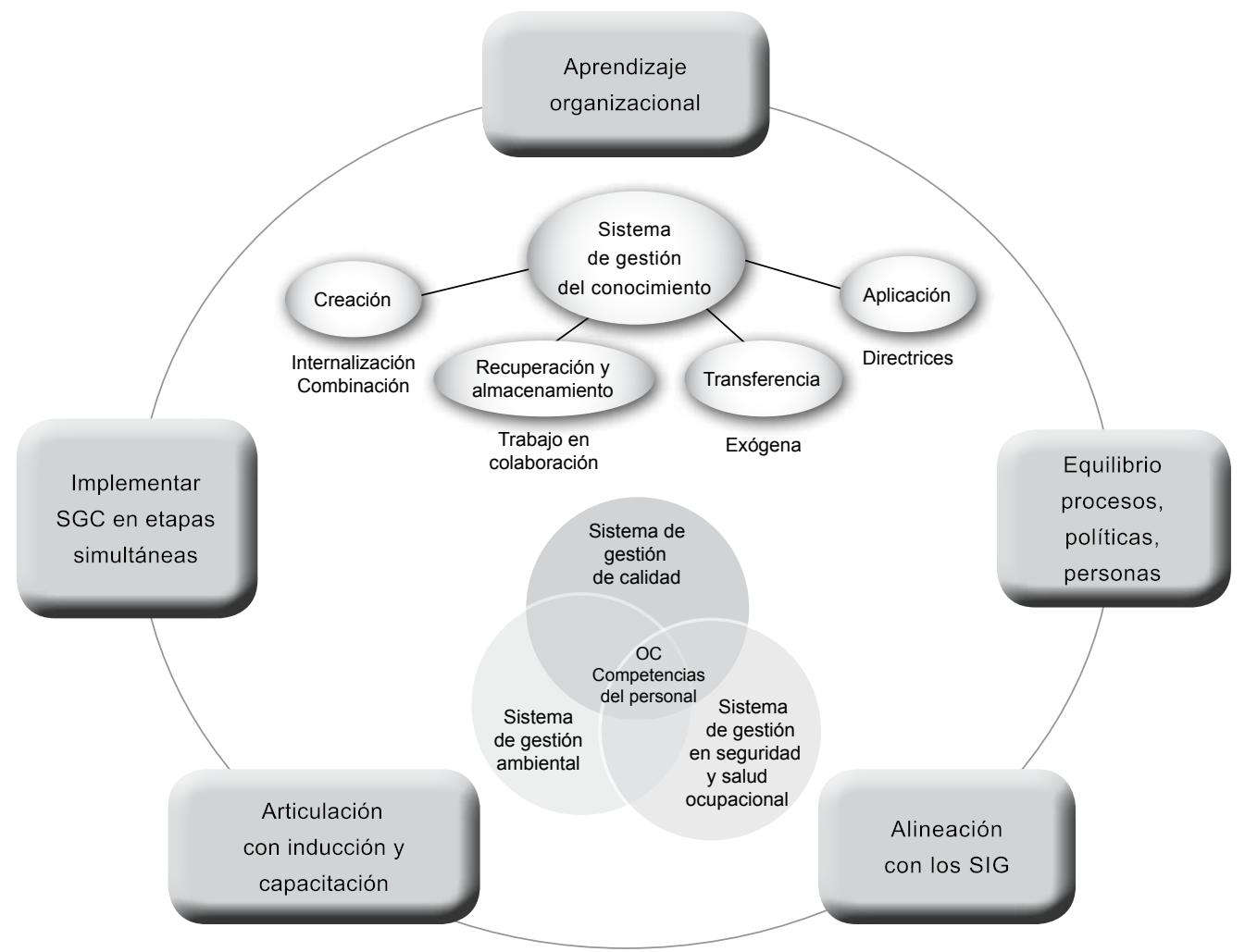

Fuente: Elaborado por las autoras con base en los resultados del estudio. 


\section{CONCLUSIONES}

- El diagnóstico refleja la situación que predomina en el Cuerpo Oficial de Bomberos frente al conocimiento; todavía este se genera a través de la experiencia del personal más antiguo de la entidad y se transfiere a los nuevos de la misma manera. Pese a lo anterior, es claro reconocer la generación del conocimiento a través de, formación formal y documentada, es decir, se valora la necesidad de cambiar hacia un conocimiento cada vez más explícito.

- A través de la implementación de un modelo de gestión del conocimiento se sientan las bases para transformar los sistemas integrados de gestión en sistemas de gestión integral, en los cuales la gestión del conocimiento, a partir de las competencias del personal apoyadas en la documentación y registros, se constituye en el elemento cohesionador 0 la ciudadanía común.

- La implementación de un modelo de gestión del conocimiento contribuye a la perdurabilidad de los sistemas de gestión, ya que aporta al cambio cultural, al aprendizaje organizacional y al mejoramiento de las competencias del personal.

- En cuanto al caso de estudio, es necesario fortalecer las competencias del personal frente al uso de las tecnologías de la información y de las comunicaciones, para desarrollar la etapa de almacenamiento y recuperación en el modelo de gestión del conocimiento. Asimismo, es necesario, en lo referente a la etapa de aplicación, en el modelo de gestión del conocimiento, intensificar el conocimiento experto a todos los niveles ya que se trata de una actividad técnica, especializada y de alto riesgo.

- Por su parte, en cuanto a la etapa transferencia del conocimiento, hay una fuerte orientación cultural a la formación externa. Sin embargo, es necesario tener mucho cuidado para no descuidar la formación interna y el fortalecimiento de las competencias del personal.

- En lo referente a la etapa de generación del conocimiento, se evidencia una fuerte tendencia a la formación formal y documentada, lo cual constituye un gran avance frente a la socialización que caracterizaba la formación bomberil.

\section{RECOMENDACIONES}

- Las organizaciones modernas deben reconocer la importancia que tiene el conocimiento y, más importante aún, su adecuada gestión en los buenos resultados que obtienen, porque es allí donde reside la verdadera ventaja competitiva y el activo más vital de las organizaciones, ya que este es lo que trasciende y perdura.

- Entidades como el Cuerpo Oficial de Bomberos de Bogotá, orientadas a la atención de emergencias, deben cambiar su estilo de trabajo y pasar de ser tan empíricos a enfocarse en el conocimiento de especialistas y de las nuevas y modernas tecnologías.

- Con el fin de evitar que los sistemas integrados de gestión, por la excesiva documentación que manejan, causen malestar en las personas, se recomienda implementar, en lo posible a la par de estos, la gestión del conocimiento de una manera adecuada, que sirva de soporte para la implementación, sin mayores traumatismos en la forma de hacer las cosas. Cuando el conocimiento se planifica y construye como eje articulador de todos los demás sistemas, como en el caso de esta investigación, permite el inicio de un proceso a través del cual se trasciende del sistema integrado de gestión al sistema de gestión integral, el cual garantiza su perdurabilidad y la verdadera interacción de cada uno de sus componentes. 
- Los datos y la información ya no son suficientes para que funcionen las entidades y las organizaciones; es fundamental transformarlos en conocimiento, que es el valor agregado necesario para la supervivencia en este mundo tan competitivo, en especial para aquellas entidades que prestan servicios.

- Es muy importante que las organizaciones, como se refleja en el modelo, entiendan que el recurso humano es lo más importante, así como la continua capacitación para fortalecer sus competencias. Es así cómo, para el caso del Cuerpo Oficial de Bomberos de Bogotá, se recomienda fortalecer su programa de capacitación en dos sentidos: en primer lugar, previo a la formulación del plan institucional de capacitación, fortalecer los conocimientos en cuanto a la gestión del conocimiento, y una vez se hayan realizado los proyectos de aprendizaje en equipo, incluir los temas pertinentes de acuerdo con el modelo establecido, los cuales aportarían para dar el paso a un modelo de gestión integral.

\section{REFERENCIAS BIBLIOGRÁFICAS}

ATENCIA, José María; GAVILÁN, Juan y RODRÍGUEZ, Agustín. Iniciación a la historia de la filosofía. 2 ed. Málaga, España: Editorial Librería Ágora, 1993. 409 p.

DAVARA, Fernando. Conocimiento. Blog sobre innovación y tecnología de tendencias. Transferencia del Conocimiento [en línea], 24 marzo de 2007. Disponible en Internet: <http://www.tendencias21. net/conocimiento/Transferencia-del-ConocimientoI_a5.html>

GRANT, Robert M. Prospering in dynamically-competitive environments: organizational capability as knowledge integration, organization science. Julio, 1996. vol. 7, no. 4, p. 375-387. Citado por LEAL MILLÁN, Antonio. Gestión del conocimiento: fundamentos y avances en investigación [presentación, en línea], [citado en 5/05/2013]. Disponible en Internet: <http:// cmapspublic. ihmc.us/rid=1173838795854_122609 230_11129/ FUNDAMENTOS.pdf >

LEAL MILLÁN, Antonio. Gestión del conocimiento: fundamentos y avances en investigación [presentación, en línea], [citado en 9/06/2013]. Disponible en Internet: $<$ http://cmapspublic.ihmc.us/rid= 1173838795854_122609230_11129/FUNDAMENTOS.pdf>

LÓPEZ LÓPEZ, Antonio José. Investigación I. Primera y segunda sesión del segundo encuentro. Cohorte 12. Maestría Calidad y Gestión Integral [presentación]. Bogotá, Colombia: Convenio USTA-Icontec, 2011.

MARTÍNEZ CARAZO, Piedad Cristina. El método de estudio de caso. Estrategia metodológica de la investigación científica. En: Pensamiento y Gestión. 2006. no. 20, p. 165-193.

MORIN, Edgar. Ciencia con conciencia. Pensamiento crítico/pensamiento utópico. Barcelona: Antrophos Editorial, 1994.369 p.

NONAKA, Ikujiro. A dynamic theory of organizational knowledge creation. En: Organization Science. Febrero, 1994. vol. 5, no. 1, p. 14-37. Citado por LEAL MILLÁN, Antonio. Gestión del conocimiento: fundamentos y avances en investigación [presentación, en línea], [citado en 15/06/2013]. Disponible en Internet: <http://cmapspublic.ihmc. us/rid=1173838795854_122609230_11129/FUNDAMENTOS.pdf> 
UCH-RRHH el portal de estudiantes de RRHH. Concepto de encuesta [en línea], 2006 [citado en 2006-06-26]. Disponible en Internet: <http:// www.gestiopolis.com/recursos/documentos/fulldocs/mar/tipencuch. htm>

\section{BIBLIOGRAFÍA}

ALCALDÍA MAYOR DE BOGOTÁ. Acuerdo 22 (9, diciembre, 1998). Por el cual se organiza el Cuerpo Oficial de Bomberos de Santafé de Bogotá, Distrito Capital, de acuerdo a lo establecido en la Ley 322 de 1996 y se dictan otras disposiciones. Registro Distrital, 1998. no. 1809.

ALCALDÍA MAYOR DE BOGOTÁ. Decreto 176 (12, mayo, 2010). Por el cual se definen los lineamientos para la conformación articulada de un sistema integrado de gestión en las entidades del Distrito Capital y se asignan unas funciones. Bogotá, 2010.

ALCALDÍA MAYOR DE BOGOTÁ. Directiva 004 (19, julio, 2005). Sistema de gestión de la calidad en las entidades y organismos distritales. Bogotá, 2005.

ANDREU, Rafael y SIEBER, Sandra. La gestión integral del conocimiento y del aprendizaje. En: Economía industrial. 1999. no. 326, p. 63-72.

BLEJMAR, Bernardo. Gestión de cambio organizacional. En: $7^{0}$ Congreso Internacional de Educación, Santillana [en línea], s. f. [citado en 2011-04-26] pp. 30-32. Disponible en Internet: <http://www. santillana.com.ar/03/congresos/7/95.pdf>

BUSTOS CLARO, Andrea Carolina; NIETO MORENO, Liliana Edith y ROJAS MANRIQUE, Leslie. Pymes colombianas y la gestión del conocimiento.
Revista Escuela de Administración de Negocios [en línea]. 2003, no. 4 [citado en 11/04/2013], p. 110-115. Disponible en Internet: <http://redalyc.uaemexmx/ src/inicio/ArtPdfRed.jsp?iCve=20604708>

CARRIÓN MAROTO, Juan. Introducción conceptual a la gestión del conocimiento [en línea], s. f. [citado en 4/05/2013]. Disponible en Internet:<http:// www.gestiondelconocimiento.com>

COLOMBIA, CONGRESO DE LA REPÚBLICA. Ley 873. (30, diciembre, 2003). Por la cual se crea el sistema de gestión de la calidad en la Rama Ejecutiva del Poder Público y en otras entidades prestadoras de servicios. Diario Oficial. Bogotá, D. C., 2004. no. 45.418.

COLOMBIA, DEPARTAMENTO NACIONAL DE PLANEACIÓN. Dirección de desarrollo empresarial. Reporte Global de Competitividad del Foro Económico Mundial 2010-2011. Resultados para Colombia 2010 [en línea]. 9, septiembre, 2010 [citado en 30/05/2013]. Disponible en Internet: $<$ http://www.dnp.gov.co/PortalWeb/LinkClick. aspx?fileticket=DzN8L_Nd9e4\%3D\&tabid=233>

DAVENPORT, Thomas H. y PRUSACK, Laurence. Working knowledge. Boston: Harvard Business School Press, 1998.

DRUCKER, Peter. El management del siglo XXI. Barcelona: Edhasa, 2000. 288 p.

INSTITUTO COLOMBIANO DE NORMAS TÉCNICAS Y CERTIFICACIÓN. Norma Técnica de Calidad de la Gestión Pública. NTCGP 1000:2009. Bogotá D.C.: El Instituto, 2009. 88 p

NONAKA, Ikujiro y TAKEUCHI, Hirotaka. The knowledge creating company. Oxford: Oxford University Press, 1995. 
PEREYRA, Beatriz. Los sistemas integrados de gestión en las organizaciones. Escuela de Administración. Informática I [en línea]. 2003, 14 p. [citado en 12/06/2013]. Disponible en Internet: <http:// www.marquetti-asociados.com.ar/down- loads/ sistemas_integrados_de_gestion.pdf>

RAMÍREZ GONZÁLEZ, Margarita. La gestión del conocimiento y su relación con la gestión de la calidad y la excelencia. Sustentación [presentación]. Bogotá, Colombia: Maestría Calidad y Gestión Integral. Convenio USTA-Icontec, 2011. Diapositiva 20.

SALAZAR CASTILLO, José Manuel y ZARANDONA AZKUENAGA, X. Análisis comparativo de las implicaciones organizativas de la calidad y de la gestión del conocimiento. En: El comportamiento de la empresa ante entornos dinámicos: XIX Congreso anual y xv Congreso Hispano Francés de AEDEM (2007) (Comunicaciones). p. 1-9.

SÁNCHEZ, M. Breve inventario de los modelos para la gestión del conocimiento en las organizaciones [en línea]. 2009 [citado en 2/06/2013]. Disponible en Internet: <http://bvs.sld.cu/revistas/ aci/vol13_6_05/aci06605.htm>

SEDEÑO PRADO, Yordan. La gestión del conocimiento, una nueva alternativa para el desarrollo de la economía latinoamericana. El caso de Cuba [en línea]. 2002 [citado en 06/06/2013]. Disponible en Internet: <http://www.gestiopolis.com/recursos/documentos/fulldocs/ger/kmcuba.htm>

SENGE, Peter. The fifth discipline. México: Random House, 1990.
SOTO BALBÓN, María Aurora y BARRIOS FERNÁNDEZ, Norma. Gestión del conocimiento. Parte I. Revisión crítica del estado del arte [en línea]. La Habana. Cuba. 30 marzo 2006. [citado en 201205-31]. Disponible en Internet: <http:// bvs.sld. cu/revistas/aci/vol14_2_06/aci04206.htm>

SVEIBY, Karl Erik. The new organizational wealth. San Francisco (CA): M Berrett-Koehler Publishers, 1997.

TORRES, Olga Lucía. Apuntes de clase. Alcaldía Mayor de Bogotá: Secretaría General, 2010.

UNIDAD ADMINISTRATIVA ESPECIAL CUERPO OFICIAL DE BOMBEROS. Estudio de clima organizacional. Informe y soportes. Bogotá, D. C., 2010.

VALOHONDO, Domingo. Gestión del conocimiento. Del mito a la realidad. Madrid: Ediciones Díaz de Santos, 2003. p. 1-56.

VESGA, Rafael. Emprendimiento e innovación en Colombia. ¿Qué nos está haciendo falta? Facultad de Administración. Universidad de los Andes. 16 p. [en línea]. [citado en 03/06/2013]. Disponible en Internet: <http://cec.uniandes. edu.co/pdf/rav.pdf>

ZAYAS RAMOS, ANA MARÍA; FRÓMETA VÁSQUEZ, Gioivys y PÉREZ MARTÍNEZ, Armenio. Los sistemas integrados de gestión. Revista Contribuciones a la Economía [en línea], mayo, 2008 [citado en 5/06/2013]. Disponible en Internet: <http://www. eumed.net/ce/2008b/ rvm.htm. ISSN:16968360> 